\title{
Strong Convergence Theorems for Nonexpansive Semigroups and Variational Inequalities in Banach Spaces
}

\author{
Haiqing Wang, ${ }^{1}$ Yongfu Su, ${ }^{1}$ and Hong Zhang ${ }^{2}$ \\ ${ }^{1}$ Department of Mathematics, Tianjin Polytechnic University, Tianjin 300160, China \\ ${ }^{2}$ Department of Mathematics, Tianjin No. 8 Middle School, Tianjin 300252, China
}

Correspondence should be addressed to Haiqing Wang, haiqingwang@tjpu.edu.cn

Received 11 November 2011; Accepted 17 December 2011

Academic Editor: Rudong Chen

Copyright (c) 2012 Haiqing Wang et al. This is an open access article distributed under the Creative Commons Attribution License, which permits unrestricted use, distribution, and reproduction in any medium, provided the original work is properly cited.

Let $X$ be a uniformly convex Banach space and $S=\{T(s): 0 \leq s<\infty\}$ be a nonexpansive semigroup such that $F(\mathcal{S})=\bigcap_{s>0} F(T(s)) \neq \emptyset$. Consider the iterative method that generates the sequence $\left\{x_{n}\right\}$ by the algorithm $x_{n+1}=\alpha_{n} f\left(x_{n}\right)+\beta_{n} x_{n}+\left(1-\alpha_{n}-\beta_{n}\right)\left(1 / s_{n}\right) \int_{0}^{s_{n}} T(s) x_{n} d s, n \geq 0$, where $\left\{\alpha_{n}\right\},\left\{\beta_{n}\right\}$, and $\left\{s_{n}\right\}$ are three sequences satisfying certain conditions, $f: C \rightarrow C$ is a contraction mapping. Strong convergence of the algorithm $\left\{x_{n}\right\}$ is proved assuming $X$ either has a weakly continuous duality map or has a uniformly Gâteaux differentiable norm.

\section{Introduction}

Let $X$ be a real Banach space and let $C$ be a nonempty closed convex subset of $X$. A mapping $T$ of $C$ into itself is said to be nonexpansive if $\|T x-T y\| \leq\|x-y\|$ for each $x, y \in C$. We denote by $F(T)$ the set of fixed points of $T$. One classical way to study nonexpansive mappings is to use contractions to approximate a nonexpansive mapping (Browder [1] and Reich [2]). More precisely, take $t \in(0,1)$ and define a contraction $T_{t}: C \rightarrow C$ by

$$
T_{t} x=t u+(1-t) T x, \quad x \in C,
$$

where $u \in C$ is a fixed point. Banach's contraction mapping principle guarantees that $T_{t}$ has a unique fixed point $x_{t}$ in $C$. It is unclear, in general, what is the behavior of $\left\{x_{t}\right\}$ as $t \rightarrow 0$, even if $T$ has a fixed point. In 1967, in the case of $T$ having a fixed point, Browder [3] proved that if $X$ is a Hilbert space, then $x_{t}$ converges strongly to the element of $F(T)$ which is nearest 
to $u$ in $F(T)$ as $t \downarrow 0$. Song and $\mathrm{Xu}[4]$ extended Browder's result to the setting of Banach spaces and proved that if $X$ is a uniformly smooth Banach space, then $x_{t}$ converges strongly to a fixed point of $T$ and the limit defines the (unique) sunny nonexpansive retraction from $C$ onto $F(T)$.

Let $f$ be a contraction on $H$ such that $\|f x-f y\| \leq \alpha\|x-y\|$, where $\alpha \in[0,1)$ is a constant. Let $x \in C, t \in(0,1)$ and $x_{t} \in C$ be the unique fixed point of the contraction $S_{t} x=t f(x)+(1-t) T x$, that is,

$$
x_{t}=t f\left(x_{t}\right)+(1-t) T x_{t} .
$$

Concerning the convergence problem of the net $\left\{x_{t}\right\}$, Moudafi [5] and Xu [6] by using the viscosity approximation method proved that the net $\left\{x_{t}\right\}$ converges strongly to a fixed point $\tilde{x}$ of $T$ in $C$ which is the unique solution to the following variational inequality:

$$
\langle(I-f) \tilde{x}, x-\tilde{x}\rangle \geq 0, \quad \forall x \in F(T) .
$$

Moreover, $\mathrm{Xu}$ [6] also studied the strong convergence of the following iterative sequence generated by

$$
x_{n+1}=\beta_{n} f\left(x_{n}\right)+\left(1-\beta_{n}\right) T x_{n}, \quad n \geq 0,
$$

where $x_{0} \in C$ is arbitrary, the sequence $\left\{\beta_{n}\right\}$ in $(0,1)$ satisfies the certain appropriate conditions.

A family $\{T(s): 0 \leq s<\infty\}$ of mappings of $C$ into itself is called a nonexpansive semigroup if it satisfies the following conditions:

(i) $T(0) x=x$ for all $x \in C$;

(ii) $T(s+t)=T(s) T(t)$ for all $x, y \in C$ and $s, t \geq 0$;

(iii) $\|T(s) x-T(s) y\| \leq\|x-y\|$ for all $x, y \in C$ and $s \geq 0$;

(iv) for all $x \in C, s \mapsto T(s) x$ is continuous.

We denote by $F(\mathcal{S})$ the set of all common fixed points of $\mathcal{S}$, that is, $F(\mathcal{S})=\{x \in C$ : $T(s) x=x, 0 \leq s<\infty\}$. It is known that $F(\mathcal{S})$ is closed and convex.

It is an interesting problem to extend above (Moudafi's [5], Xu's [6], and so on) results to the nonexpansive semigroup case. Recently, for the nonexpansive semigroups $\mathcal{S}=\{T(s)$ : $0 \leq s<\infty\}$, Plubtieng and Punpaeng [7] studied the continuous scheme $\left\{x_{t}\right\}$ defined by

$$
x_{t}=t f\left(x_{t}\right)+(1-t) \frac{1}{\lambda_{t}} \int_{0}^{\lambda_{t}} T(s) x_{t} d s,
$$

where $t \in(0,1)$ and $\left\{\lambda_{t}\right\}$ is a positive real divergent net, and the iterative scheme $\left\{x_{n}\right\}$ defined by

$$
x_{n+1}=\alpha_{n} f\left(x_{n}\right)+\beta_{n} x_{n}+\left(1-\alpha_{n}-\beta_{n}\right) \frac{1}{s_{n}} \int_{0}^{s_{n}} T(s) x_{n} d s, \quad n \geq 0
$$


where $x_{0} \in C,\left\{\alpha_{n}\right\},\left\{\beta_{n}\right\}$ are a sequence in $(0,1)$ and $\left\{s_{n}\right\}$ is a positive real divergent real sequence in the setting of a real Hilbert space. They proved the continuous scheme $\left\{x_{t}\right\}$ defined by (1.5) and the iterative scheme $\left\{x_{n}\right\}$ defined by (1.6) converge strongly to a fixed point $\tilde{x}$ of $\mathcal{S}$ which is the unique solution of the variational inequality (1.3). At this stage, the following question arises naturally.

Question 1. Do Plubtieng and Punpaeng's results hold for the nonexpansive semigroups in a Banach space?

The purpose of this paper is to give affirmative answers of Question 1. One result of this paper says that Plubtieng and Punpaeng's results hold in a uniformly convex Banach space which has a weakly continuous duality map.

On the other hand, Chen and Song [8] proved the following implicit and explicit viscosity iteration processes defined by (1.7) to nonexpansive semigroup case,

$$
\begin{aligned}
& x_{n}=\alpha_{n} f\left(x_{n}\right)+\left(1-\alpha_{n}\right) \frac{1}{s_{n}} \int_{0}^{s_{n}} T(s) x_{n} d s, \quad n \geq 0, \\
& x_{n+1}=\alpha_{n} f\left(x_{n}\right)+\left(1-\alpha_{n}\right) \frac{1}{s_{n}} \int_{0}^{s_{n}} T(s) x_{n} d s, \quad n \geq 0 .
\end{aligned}
$$

And they proved that $\left\{x_{n}\right\}$ converges strongly to a common fixed point of $F(\mathcal{S})$ in a uniformly convex Banach space with a uniformly Gâteaux differentiable norm.

Motivated by the above results, the other result of this paper says that Plubtieng and Punpaeng's results hold in the framework of uniformly convex Banach space with a uniformly Gâteaux differentiable norm. The results improve and extend the corresponding results of Plubtieng and Punpaeng [7], Chen and Song [8], Moudafi's [5], Xu's [6], and others.

\section{Preliminaries}

Let $X$ be a real Banach space with inner product $\langle\cdot, \cdot\rangle$ and norm $\|\cdot\|$, respectively. Let $J$ denote the normalized duality mapping from $X$ into the dual space $2^{X^{*}}$ given by

$$
J(x)=\left\{x^{*} \in X^{*}:\left\langle x, x^{*}\right\rangle=\|x\|^{2}=\left\|x^{*}\right\|^{2}\right\}, \quad x \in X .
$$

In the sequel, we will denote the single valued duality mapping by $j$. When $\left\{x_{n}\right\}$ is a sequence in $X$, then $x_{n} \rightarrow x\left(x_{n} \rightarrow x\right)$ will denote strong (weak) convergence of the sequence $\left\{x_{n}\right\}$ to $x$.

Let $S(X)=\{x \in X:\|x\|=1\}$. Then the norm of $X$ is said to be Gateaux differentiable if

$$
\lim _{t \rightarrow 0} \frac{\|x+t y\|-\|x\|}{t}
$$

exists for each $x, y \in S(X)$. In this case, $X$ is called smooth. The norm of $X$ is said to be uniformly Gateaux differentiable if for each $y \in S(X)$, the limit (2.2) is attained uniformly for $x \in S(X)$. It is well known that $X$ is smooth if and only if any duality mapping on $X$ is sigle valued. 
Also if $X$ has a uniformly Gâteaux differentiable norm, then the duality mapping is normto-weak* uniformly continuous on bounded sets. The norm of $E$ is called Fréchet differentiable, if for each $x \in S(X)$, the limit (2.2) is attained uniformly for $y \in S(X)$. The norm of $X$ is called uniformly Fréchet differentiable, if the limit (2.2) is attained uniformly for $x, y \in S(X)$. It is well known that (uniformly) Fréchet differentiability of the norm of $X$ implies (uniformly) Gâteaux differentiability of the norm of $X$ and $X$ is uniformly smooth if and only if the norm of $X$ is uniformly Fréchet differentiable.

A Banach space $X$ is said to be strictly convex if

$$
\|x\|=\|y\|=1, \quad x \neq y \text { implies } \frac{\|x+y\|}{2}<1 .
$$

A Banach space $X$ is said to be uniformly convex if $\delta_{X}(\varepsilon)>0$ for all $\varepsilon>0$, where $\delta_{X}(\varepsilon)$ is modulus of convexity of $E$ defined by

$$
\delta_{E}(\varepsilon)=\inf \left\{1-\frac{\|x+y\|}{2}:\|x\| \leq 1,\|y\| \leq 1,\|x-y\| \geq \varepsilon\right\}, \quad \varepsilon \in[0,2]
$$

A uniformly convex Banach space $E$ is reflexive and strictly convex [9, Theorem 4.1.6, Theorem 4.1.2].

Lemma 2.1 (Goebel and Reich [10], Proposition 5.3). Let $C$ be a nonempty closed convex subset of a strictly convex Banach space $X$ and $T: C \rightarrow C$ a nonexpansive mapping with $F(T) \neq \emptyset$. Then $F(T)$ is closed and convex.

Lemma 2.2 (see Xu [11]). In a smooth Banach space X there holds the inequality

$$
\|x+y\|^{2} \leq\|x\|^{2}+2\langle y, J(x+y)\rangle, \quad x, y, \in X
$$

Lemma 2.3 (Browder [12]). Let E be a uniformly convex Banach space, K a nonempty closed convex subset of $E$, and $T: K \rightarrow E$ a nonexpansive mapping. Then $I-T$ is demi closed at zero.

Lemma 2.4 (see [8, Lemma 2.7]). Let C be a nonempty bounded closed convex subset of a uniformly convex Banach space $X$, and let $\mathcal{S}=\{T(s): 0 \leq s<\infty\}$ be a nonexpansive semigroup on $C$ such that $F(S) \neq \emptyset$. For $x \in C$ and $t>0$. Then, for any $0 \leq h<\infty$,

$$
\limsup _{t \rightarrow \infty}\left\|\frac{1}{t} \int_{0}^{t} T(s) x d s-T(h)\left(\frac{1}{t} \int_{0}^{t} T(s) x d s\right)\right\|=0 .
$$

Recall that a gauge is a continuous strictly increasing function $\varphi:[0, \infty) \rightarrow[0, \infty)$ such that $\varphi(0)=0$ and $\varphi(t) \rightarrow \infty$ as $t \rightarrow \infty$. Associated to a gauge $\varphi$ is the duality map $J_{\varphi}: X \rightarrow X^{*}$ defined by

$$
J_{\varphi}(x)=\left\{x^{*} \in X^{*}:\left\langle x, x^{*}\right\rangle=\|x\| \varphi(\|x\|),\left\|x^{*}\right\|=\varphi(\|x\|)\right\}, \quad x \in X .
$$


Following Browder [13], we say that a Banach space $X$ has a weakly continuous duality map if there exists a gauge $\varphi$ for which the duality map $J_{\varphi}$ is single valued and weak-to-weak ${ }^{*}$ sequentially continuous (i.e., if $\left\{x_{n}\right\}$ is a sequence in $X$ weakly convergent to a point $x$, then the sequence $J_{\varphi}\left(x_{n}\right)$ converges weakly* to $\left.J_{\varphi}(x)\right)$. It is known that $l^{p}$ has a weakly continuous duality map for all $1<p<\infty$. Set

$$
\Phi(t)=\int_{0}^{t} \varphi(\tau) d \tau, \quad t \geq 0
$$

Then

$$
J_{\varphi}(x)=\partial \Phi(\|x\|), \quad x \in X,
$$

where $\partial$ denotes the subdifferential in the sense of convex analysis. The next lemma is an immediate consequence of the subdifferential inequality.

Lemma 2.5 (Xu [11, Lemma 2.6]). Assume that $X$ has a weakly continuous duality map $J_{\varphi}$ with gauge $\varphi$, for all $x, y \in X$, there holds the inequality

$$
\Phi(\|x+y\|) \leq \Phi(\|x\|)+\left\langle y, J_{\varphi}(x+y)\right\rangle .
$$

Lemma 2.6 (Xu [6]). Assume $\left\{\alpha_{n}\right\}$ is a sequence of nonnegative real numbers such that

$$
\alpha_{n+1} \leq\left(1-\gamma_{n}\right) \alpha_{n}+\delta_{n}, \quad n \geq 0,
$$

where $\left\{\gamma_{n}\right\}$ is a sequence in $(0,1)$ and $\left\{\delta_{n}\right\}$ is a sequence in $\mathbf{R}$ such that

(i) $\sum_{n=1}^{\infty} \gamma_{n}=\infty$;

(ii) $\lim \sup _{n \rightarrow \infty} \delta_{n} / \gamma_{n} \leq 0$ or $\sum_{n=1}^{\infty}\left|\delta_{n}\right|<\infty$.

Then $\lim _{n \rightarrow \infty} \alpha_{n}=0$.

Finally, we also need the following definitions and results $[9,14]$. Let $\mu$ be a continuous linear functional on $l^{\infty}$ satisfying $\|\mu\|=1=\mu(1)$. Then we know that $\mu$ is a mean on $N$ if and only if

$$
\inf \left\{a_{n} ; n \in N\right\} \leq \mu(a) \leq \sup \left\{a_{n} ; n \in N\right\},
$$

for every $a=\left(a_{1}, a_{2}, \ldots\right) \in l^{\infty}$. Occasionally, we will use $\mu_{n}\left(a_{n}\right)$ instead of $\mu(a)$. A mean $\mu$ on $N$ is called a Banach limit if

$$
\mu_{n}\left(a_{n}\right)=\mu_{n}\left(a_{n+1}\right),
$$


for every $a=\left(a_{1}, a_{2}, \ldots\right) \in l^{\infty}$. Using the Hahn-Banach theorem, or the Tychonoff fixed point theorem, we can prove the existence of a Banach limit. We know that if $\mu$ is a Banach limit, then

$$
\liminf _{n \rightarrow \infty} a_{n} \leq \mu_{n}\left(a_{n}\right) \leq \limsup _{n \rightarrow \infty} a_{n}
$$

for every $a=\left(a_{1}, a_{2}, \ldots\right) \in l^{\infty}$. So, if $a=\left(a_{1}, a_{2}, \ldots\right), b=\left(b_{1}, b_{2}, \ldots\right) \in l^{\infty}$, and $a_{n} \rightarrow c$ (resp., $\left.a_{n}-b_{n} \rightarrow 0\right)$, as $n \rightarrow \infty$, we have

$$
\mu_{n}\left(a_{n}\right)=\mu(a)=c\left(\text { resp., } \mu_{n}\left(a_{n}\right)=\mu_{n}\left(b_{n}\right)\right) .
$$

Subsequently, the following result was showed in [14, Lemma 1] and [9, Lemma 4.5.4].

Lemma 2.7 (see [14, Lemma 1]). Let $C$ be a nonempty closed convex subset of a Banach space X with a uniformly Gâteaux differentiable norm and $\left\{x_{n}\right\}$ a bounded sequence of $E$. If $z_{0} \in C$, then

$$
\mu_{n}\left\|x_{n}-z_{0}\right\|^{2}=\min _{x \in C} \mu_{n}\left\|x_{n}-x\right\|^{2}
$$

if and only if

$$
\mu_{n}\left\langle x-z_{0}, J\left(x_{n}-z_{0}\right)\right\rangle \leq 0, \quad \forall x \in C
$$

Lemma 2.8 (Song and $\mathrm{Xu}$ [4, Proposition 3.1]). Let X be a reflexive strictly convex Banach space with a uniformly Gateaux differentiable norm, and $C$ a nonempty closed convex subset of $X$. Suppose $\left\{x_{n}\right\}$ is a bounded sequence in $C$ such that $\lim _{n \rightarrow \infty}\left\|x_{n}-T x_{n}\right\|=0$, an approximate fixed point of nonexpansive self-mapping $T$ on $C$. Define the set

$$
C^{*}=\left\{y \in C: \mu_{n}\left\|x_{n}-y\right\|^{2}=\inf _{x \in C} \mu_{n}\left\|x_{n}-x\right\|^{2}\right\}
$$

If $F(T) \neq \emptyset$, then $C^{*} \cap F(T) \neq \emptyset$.

\section{Implicit Iteration Scheme}

Theorem 3.1. Let X be a uniformly convex Banach space that has a weakly continuous duality map $J_{\varphi}$ with gauge $\varphi$, and let $C$ be a nonempty closed convex subset of $X$. Let $\mathcal{S}=\{T(s): 0 \leq s<\infty\}$ be a nonexpansive semigroup from $C$ into itself such that $F(\mathcal{S})=\bigcap_{s>0} F(T(s)) \neq \emptyset$ and $f: C \rightarrow C$ a contraction mapping with the contractive coefficient $\alpha \in[0,1)$. Suppose $\left\{\lambda_{t}\right\}_{0<t<1}$ is a net of positive real numbers such that $\lim _{t \rightarrow 0^{+}} \lambda_{t}=\infty$, the sequence $\left\{x_{t}\right\}$ is given by the following equation:

$$
x_{t}=t f\left(x_{t}\right)+(1-t) \frac{1}{\lambda_{t}} \int_{0}^{\lambda_{t}} T(s) x_{t} d s
$$


Then $\left\{x_{t}\right\}$ converges strongly to $\tilde{x}$ as $t \rightarrow 0^{+}$, where $\tilde{x}$ is the unique solution in $F(\mathcal{S})$ of the variational inequality

$$
\langle(I-f) \tilde{x}, J(x-\tilde{x})\rangle \geq 0, \quad x \in F(\mathcal{S}) .
$$

Proof. Note that $F(S)$ is a nonempty closed convex set by Lemma 2.1. We first show that $\left\{x_{t}\right\}$ is bounded. Indeed, for any fixed $p \in F(\mathcal{S})$, we have

$$
\begin{aligned}
\left\|x_{t}-p\right\| & \leq t\left\|f\left(x_{t}\right)-p\right\|+(1-t)\left\|\frac{1}{\lambda_{t}} \int_{0}^{\lambda_{t}} T(s) x_{t} d s-p\right\| \\
& \leq t\left(\left\|f\left(x_{t}\right)-f(p)\right\|+\|f(p)-p\|\right)+(1-t) \frac{1}{\lambda_{t}} \int_{0}^{\lambda_{t}}\left\|T(s) x_{t}-p\right\| d s \\
& \leq t\left(\alpha\left\|x_{t}-p\right\|+\|f(p)-p\|\right)+(1-t)\left\|x_{t}-p\right\| \\
& =\left\|x_{t}-p\right\|-t(1-\alpha)\left\|x_{t}-p\right\|+t\|f(p)-p\| .
\end{aligned}
$$

It follows that

$$
\left\|x_{t}-p\right\| \leq \frac{1}{1-\alpha}\|f(p)-p\|
$$

Thus $\left\{x_{t}\right\}$ is bounded, so are $\left\{f\left(x_{t}\right)\right\}$ and $\left\{T(s) x_{t}\right\}$ for every $0 \leq s<\infty$. Furthermore, we note that

$$
\begin{aligned}
\left\|x_{t}-T(s) x_{t}\right\| \leq & \left\|x_{t}-\frac{1}{\lambda_{t}} \int_{0}^{\lambda_{t}} T(s) x_{t} d s\right\|+\left\|\frac{1}{\lambda_{t}} \int_{0}^{\lambda_{t}} T(s) x_{t} d s-T(s)\left(\frac{1}{\lambda_{t}} \int_{0}^{\lambda_{t}} T(s) x_{t} d s\right)\right\| \\
& +\left\|T(s)\left(\frac{1}{\lambda_{t}} \int_{0}^{\lambda_{t}} T(s) x_{t} d s\right)-T(s) x_{t}\right\| \\
& \leq 2\left\|x_{t}-\frac{1}{\lambda_{t}} \int_{0}^{\lambda_{t}} T(s) x_{t} \mathrm{~d} s\right\|+\left\|\frac{1}{\lambda_{t}} \int_{0}^{\lambda_{t}} T(s) x_{t} d s-T(s)\left(\frac{1}{\lambda_{t}} \int_{0}^{\lambda_{t}} T(s) x_{t} d s\right)\right\|,
\end{aligned}
$$

for every $0 \leq s<\infty$. On the one hand, we observe that

$$
\left\|x_{t}-\frac{1}{\lambda_{t}} \int_{0}^{\lambda_{t}} T(s) x_{t} d s\right\|=\frac{t}{1-t}\left\|x_{t}-f\left(x_{t}\right)\right\|,
$$


for every $t>0$. On the other hand, let $z_{0} \in F(S)$ and $D=\left\{z \in C:\left\|z-z_{0}\right\| \leq\left\|f\left(z_{0}\right)-z_{0}\right\|\right\}$, then $D$ is a nonempty closed bounded convex subset of $C$ which is $T(s)$-invariant for each $0 \leq s<\infty$ and contains $\left\{x_{t}\right\}$. It follows by Lemma 2.4 that

$$
\begin{aligned}
\lim _{\lambda_{t} \rightarrow \infty} & \left\|\frac{1}{\lambda_{t}} \int_{0}^{\lambda_{t}} T(s) x_{t} d s-T(\mathrm{~s})\left(\frac{1}{\lambda_{t}} \int_{0}^{\lambda_{t}} T(s) x_{t} d s\right)\right\| \\
& \leq \lim _{\lambda_{t} \rightarrow \infty} \sup _{x \in D}\left\|\frac{1}{\lambda_{t}} \int_{0}^{\lambda_{t}} T(s) x_{t} d s-T(s)\left(\frac{1}{\lambda_{t}} \int_{0}^{\lambda_{t}} T(s) x_{t} d s\right)\right\|=0 .
\end{aligned}
$$

Hence, by (3.5)-(3.7), we obtain

$$
\left\|x_{t}-T(s) x_{t}\right\| \longrightarrow 0 \quad \text { as } t \longrightarrow 0
$$

for every $0 \leq s<\infty$. Assume $\left\{t_{n}\right\}_{n=1}^{\infty} \subset(0,1)$ is such that $t_{n} \rightarrow 0$ as $n \rightarrow \infty$. Put $x_{n}:=x_{t_{n}}$, $\lambda_{n}:=\lambda_{t_{n}}$, we will show that $\left\{x_{n}\right\}$ contains s subsequence converging strongly to $\tilde{x}$, where $\tilde{x} \in F(\mathcal{S})$. Since $\left\{x_{n}\right\}$ is a bounded sequence, there is a subsequence $\left\{x_{n_{j}}\right\}$ of $\left\{x_{n}\right\}$ which converges weakly to $\tilde{x} \in C$. By Lemma 2.3, we have $\tilde{x} \in F(\mathcal{S})$. For each $n \geq 1$, we have

$$
x_{n}-\tilde{x}=t_{n}\left(f\left(x_{n}\right)-\tilde{x}\right)+\left(1-t_{n}\right)\left(\frac{1}{\lambda_{n}} \int_{0}^{\lambda_{n}} T(s) x_{n} d s-\tilde{x}\right) .
$$

Thus, by Lemma 2.5 , we obtain

$$
\begin{aligned}
\Phi\left(\left\|x_{n}-\tilde{x}\right\|\right) & =\Phi\left(\left\|t_{n}\left(f\left(x_{n}\right)-\tilde{x}\right)+\left(1-t_{n}\right)\left(\frac{1}{\lambda_{n}} \int_{0}^{\lambda_{n}} T(s) x_{n} d s-\tilde{x}\right)\right\|\right) \\
& \leq \Phi\left(\left\|\left(1-t_{n}\right)\left(\frac{1}{\lambda_{n}} \int_{0}^{\lambda_{n}} T(s) x_{n} d s-\tilde{x}\right)\right\|\right)+t_{n}\left\langle f\left(x_{n}\right)-\tilde{x}, J_{\varphi}\left(x_{n}-\tilde{x}\right)\right\rangle \\
& \leq\left(1-t_{n}\right) \Phi\left(\left\|\frac{1}{\lambda_{n}} \int_{0}^{\lambda_{n}} T(s) x_{n} d s-\tilde{x}\right\|\right)+t_{n}\left\langle f\left(x_{n}\right)-\tilde{x}, J_{\varphi}\left(x_{n}-\tilde{x}\right)\right\rangle \\
& \leq\left(1-t_{n}\right) \Phi\left(\left\|x_{n}-\tilde{x}\right\|\right)+t_{n}\left\langle f\left(x_{n}\right)-\tilde{x}, J_{\varphi}\left(x_{n}-\tilde{x}\right)\right\rangle .
\end{aligned}
$$

This implies that

$$
\Phi\left(\left\|x_{n}-\tilde{x}\right\|\right) \leq\left\langle f\left(x_{n}\right)-\tilde{x}, J_{\varphi}\left(x_{n}-\tilde{x}\right)\right\rangle .
$$


In particular, we have

$$
\Phi\left(\left\|x_{n_{j}}-\tilde{x}\right\|\right) \leq\left\langle f\left(x_{n_{j}}\right)-\tilde{x}, J_{\varphi}\left(x_{n_{j}}-\tilde{x}\right)\right\rangle .
$$

Now observing that $\left\{x_{n_{j}}\right\} \rightarrow \tilde{x}$ implies $J_{\varphi}\left(x_{n_{j}}-\tilde{x}\right) \rightarrow 0$. And since $f\left(x_{n_{j}}\right)$ is bounded, it follows from (3.12) that

$$
\Phi\left(\left\|x_{n_{j}}-\tilde{x}\right\|\right) \longrightarrow 0 \quad \text { as } j \longrightarrow \infty
$$

Hence $x_{n_{j}} \rightarrow \tilde{x}$.

Next, we show that $\tilde{x} \in F(\mathcal{S})$ solves the variational inequality (3.2). Indeed, for $q \in$ $F(\mathcal{S})$, it is easy to see that

$$
\begin{aligned}
\left\langle x_{t}-\frac{1}{\lambda_{t}} \int_{0}^{\lambda_{t}} T(s) x_{t} d s, J_{\varphi}\left(x_{t}-q\right)\right\rangle & =\Phi\left(\left\|x_{t}-q\right\|\right)+\left\langle q-\frac{1}{\lambda_{t}} \int_{0}^{\lambda_{t}} T(s) x_{t} d s, J_{\varphi}\left(x_{t}-q\right)\right\rangle \\
& \geq \Phi\left(\left\|x_{t}-q\right\|\right)-\left\|q-\frac{1}{\lambda_{t}} \int_{0}^{\lambda_{t}} T(s) x_{t} d s\right\|\left\|J_{\varphi}\left(x_{t}-q\right)\right\| \\
& \geq \Phi\left(\left\|x_{t}-q\right\|\right)-\Phi\left(\left\|x_{t}-q\right\|\right) \\
& =0 .
\end{aligned}
$$

However, we note that

$$
x_{t}-\frac{1}{\lambda_{t}} \int_{0}^{\lambda_{t}} T(s) x_{t} d s=\frac{t}{1-t}\left(f\left(x_{t}\right)-x_{t}\right) .
$$

Thus, we get that for $t \in(0,1)$ and $q \in F(\mathcal{S})$

$$
\left\langle x_{t}-f\left(x_{t}\right), J_{\varphi}\left(x_{t}-q\right)\right\rangle \leq 0 .
$$

Taking the limit through $t:=t_{n_{j}} \rightarrow 0$, we obtain

$$
\left\langle(I-f) \tilde{x}, J_{\varphi}(\tilde{x}-q)\right\rangle \leq 0, \quad \forall q \in F(\mathcal{S}) .
$$


This implies that

$$
\langle(I-f) \tilde{x}, J(\tilde{x}-q)\rangle \leq 0, \quad \forall q \in F(\mathcal{S}),
$$

since $J_{\varphi}(x)=(\varphi(\|x\|) /\|x\|) J(x)$ for $x \neq 0$.

Finally, we show that the net $\left\{x_{t}\right\}$ convergence strong to $\tilde{x}$. Assume that there is a sequence $\left\{s_{n}\right\} \subset(0,1)$ such that $x_{s_{n}} \rightarrow \bar{x}$, where $s_{n} \rightarrow 0$. we note by Lemma 2.3 that $\bar{x} \in F(\mathcal{S})$. It follows from the inequality (3.18) that

$$
\langle(I-f) \tilde{x}, J(\tilde{x}-\bar{x})\rangle \leq 0
$$

Interchange $\tilde{x}$ and $\bar{x}$ to obtain

$$
\langle(I-f) \bar{x}, J(\bar{x}-\tilde{x})\rangle \leq 0 \text {. }
$$

Adding (3.19) and (3.20) yields

$$
(1-\alpha)\|\tilde{x}-\bar{x}\|^{2} \leq\langle\bar{x}-\tilde{x}-(f(\tilde{x})-f(\bar{x})), J(\bar{x}-\tilde{x})\rangle \leq 0 .
$$

We must have $\tilde{x}=\bar{x}$ and the uniqueness is proved. In a summary, we have shown that each cluster point of $\left\{x_{t}\right\}$ as $t \rightarrow 0$ equals $\tilde{x}$. Therefore $x_{t} \rightarrow \tilde{x}$ as $t \rightarrow 0$.

Theorem 3.2. Let $X$ be a uniformly convex Banach space with a uniformly Gateaux differentiable norm and $C$ be a nonempty closed convex subset of $X$. Let $\mathcal{S}=\{T(s): 0 \leq s<\infty\}$ be a nonexpansive semigroup from $C$ into itself such that $F(\mathcal{S})=\bigcap_{s>0} F(T(s)) \neq \emptyset$ and $f: C \rightarrow C$ a contraction mapping with the contractive coefficient $\alpha \in[0,1)$. Suppose $\left\{\lambda_{t}\right\}_{0<t<1}$ is a net of positive real numbers such that $\lim _{t \rightarrow 0^{+}} \lambda_{t}=\infty$, the sequence $\left\{x_{t}\right\}$ is given by the following equation:

$$
x_{t}=t f\left(x_{t}\right)+(1-t) \frac{1}{\lambda_{t}} \int_{0}^{\lambda_{t}} T(s) x_{t} d s
$$

Then $\left\{x_{t}\right\}$ converges strongly to $\tilde{x}$ as $t \rightarrow 0^{+}$, where $\tilde{x}$ is the unique solution in $F(\mathcal{S})$ of the variational inequality

$$
\langle(I-f) \tilde{x}, J(x-\tilde{x})\rangle \geq 0, \quad x \in F(\mathcal{S}) .
$$

Proof. We include only those points in this proof which are different from those already presented in the proof of Theorem 3.1. As in the proof of Theorem 3.1, we obtain that there is a subsequence $\left\{x_{n_{j}}\right\}$ of $\left\{x_{n}\right\}$ which converges weakly to $\tilde{x} \in F(\mathcal{S})$. For each $n \geq 1$, we have

$$
x_{n}-\tilde{x}=t_{n}\left(f\left(x_{n}\right)-\tilde{x}\right)+\left(1-t_{n}\right)\left(\frac{1}{\lambda_{n}} \int_{0}^{\lambda_{n}} T(s) x_{n} d s-\tilde{x}\right) .
$$


Thus, we have

$$
\begin{aligned}
\left\|x_{n}-\tilde{x}\right\|^{2}= & \left\langle t_{n}\left(f\left(x_{n}\right)-\tilde{x}\right)+\left(1-t_{n}\right)\left(\frac{1}{\lambda_{n}} \int_{0}^{\lambda_{n}} T(s) x_{n} d s-\tilde{x}\right), J\left(x_{n}-\tilde{x}\right)\right\rangle \\
= & t_{n}\left\langle f\left(x_{n}\right)-f(\tilde{x})+f(\tilde{x})-\tilde{x}, J\left(x_{n}-\tilde{x}\right)\right\rangle \\
& +\left(1-t_{n}\right)\left\langle\left(\frac{1}{\lambda_{n}} \int_{0}^{\lambda_{n}} T(s) x_{n} d s-\tilde{x}\right), J\left(x_{n}-\tilde{x}\right)\right\rangle \\
\leq & t_{n}\left\|f\left(x_{n}\right)-f(\tilde{x})\right\|\left\|J\left(x_{n}-\tilde{x}\right)\right\|+t_{n}\left\langle f(\tilde{x})-\tilde{x}, J\left(x_{n}-\tilde{x}\right)\right\rangle \\
& +\left(1-t_{n}\right)\left\|\frac{1}{\lambda_{n}} \int_{0}^{\lambda_{n}} T(s) x_{n} d s-\tilde{x}\right\|\left\|J\left(x_{n}-\tilde{x}\right)\right\| \\
\leq & \left(1-(1-\alpha) t_{n}\right)\left\|x_{n}-\tilde{x}\right\|^{2}+t_{n}\left\langle f(\tilde{x})-\tilde{x}, J\left(x_{n}-\tilde{x}\right)\right\rangle .
\end{aligned}
$$

Therefore,

$$
\left\|x_{n}-\tilde{x}\right\|^{2} \leq \frac{1}{1-\alpha}\left\langle f(\tilde{x})-\tilde{x}, J\left(x_{n}-\tilde{x}\right)\right\rangle
$$

We claim that the set $\left\{x_{n}\right\}$ is sequentially compact. Indeed, define the set

$$
C^{*}=\left\{y \in C: \mu_{n}\left\|x_{n}-y\right\|^{2}=\inf _{x \in C} \mu_{n}\left\|x_{n}-x\right\|^{2}\right\}
$$

By Lemma 2.8, we found $\tilde{x} \in C^{*}$. Using Lemma 2.7 we get that

$$
\mu_{n}\left\langle x-\tilde{x}, J\left(x_{n}-\tilde{x}\right)\right\rangle \leq 0, \quad \forall x \in C .
$$

From (3.26), we get

$$
\mu_{n}\left\|x_{n}-\tilde{x}\right\|^{2} \leq \frac{1}{1-\alpha} \mu_{n}\left\langle f(\tilde{x})-\tilde{x}, J\left(x_{n}-\tilde{x}\right)\right\rangle \leq 0,
$$

that is

$$
\mu_{n}\left\|x_{n}-\tilde{x}\right\|=0
$$

Hence, there exists a subsequence $\left\{x_{n_{k}}\right\}$ of $\left\{x_{n}\right\}$ converges strongly to $\tilde{x} \in F(\mathcal{S})$ as $k \rightarrow \infty$. 
Next we show that $\tilde{x}$ is a solution in $F(S)$ to the variational inequality (3.23). In fact, for any fixed $x \in F(\mathcal{S})$, there exists a constant $M>0$ such that $\left\|x_{n}-x\right\| \leq M$, then

$$
\begin{aligned}
\left\|x_{n}-x\right\|^{2}= & t_{n}\left\langle f\left(x_{n}\right)-f(\tilde{x})+\tilde{x}-x_{n}, J\left(x_{n}-x\right)\right\rangle+t_{n}\left\langle f(\tilde{x})-\tilde{x}, J\left(x_{n}-x\right)\right\rangle \\
& +t_{n}\left\langle x_{n}-x, J\left(x_{n}-x\right)\right\rangle+\left(1-t_{n}\right)\left\langle\frac{1}{\lambda_{n}} \int_{0}^{\lambda_{n}} T(s) x_{n} d s-x, J\left(x_{n}-x\right)\right\rangle \\
\leq & (1+\alpha) t_{n} M\left\|x_{n}-\tilde{x}\right\|+t_{n}\left\langle f(\tilde{x})-\tilde{x}, J\left(x_{n}-x\right)\right\rangle+\left\|x_{n}-x\right\|^{2} .
\end{aligned}
$$

Therefore,

$$
\left\langle f(\tilde{x})-\tilde{x}, J\left(x-x_{n}\right)\right\rangle \leq(1+\alpha) M\left\|x_{n}-\tilde{x}\right\| .
$$

Since the duality mapping $J$ is single valued and norm topology to weak* topology uniformly continuous on any bounded subset of a Banach space X with a uniformly Gâteaux differentiable norm, we have

$$
\left\langle f(\tilde{x})-\tilde{x}, J\left(x-x_{n_{k}}\right)\right\rangle \longrightarrow\langle f(\tilde{x})-\tilde{x}, J(x-\tilde{x})\rangle .
$$

Taking limit as $j \rightarrow \infty$ in two sides of (3.32), we get

$$
\langle f(\tilde{x})-\tilde{x}, J(x-\tilde{x})\rangle \leq 0, \quad \forall x \in F(\mathcal{S}) .
$$

Finally we will show that the net $\left\{x_{t}\right\}$ convergence strong to $\tilde{x}$. This section is similar to that of Theorem 3.1.

\section{Explicit Iterative Scheme}

Theorem 4.1. Let $X$ be a uniformly convex Banach space that has a weakly continuous duality map $J_{\varphi}$ with gauge $\varphi$ and $C$ be a nonempty closed convex subset of $X$. Let $\mathcal{S}=\{T(s): 0 \leq s<\infty\}$ be a nonexpansive semigroup from $C$ into itself such that $F(\mathcal{S})=\bigcap_{s>0} F(T(s)) \neq \emptyset$ and $f: C \rightarrow C$ a contraction mapping with the contractive coefficient $\alpha \in[0,1)$. Let $\left\{\alpha_{n}\right\}$ and $\left\{\beta_{n}\right\}$ be the sequence in $(0,1)$ which satisfies $\alpha_{n}+\beta_{n}<1, \lim _{n \rightarrow \infty} \alpha_{n} \rightarrow 0, \lim _{n \rightarrow \infty} \beta_{n} \rightarrow 0$ and $\sum_{n=1}^{\infty} \alpha_{n}=\infty$, and $\left\{s_{n}\right\}$ is a positive real divergent sequence such that $\lim _{n \rightarrow \infty} s_{n} \rightarrow \infty$. If the sequence $\left\{x_{n}\right\}$ defined by $x_{0} \in C$ and

$$
x_{n+1}=\alpha_{n} f\left(x_{n}\right)+\beta_{n} x_{n}+\left(1-\alpha_{n}-\beta_{n}\right) \frac{1}{s_{n}} \int_{0}^{s_{n}} T(s) x_{n} d s, \quad n \geq 0 .
$$

Then $\left\{x_{n}\right\}$ converges strongly to $\tilde{x}$ as $n \rightarrow \infty$, where $\tilde{x}$ is the unique solution in $F(\mathcal{S})$ of the variational inequality

$$
\langle(I-f) \tilde{x}, J(x-\tilde{x})\rangle \geq 0, \quad x \in F(\mathcal{S}) .
$$


Journal of Applied Mathematics

Proof. Note that $F(\mathcal{S})$ is a nonempty closed convex set. We first show that $\left\{x_{n}\right\}$ is bounded. Let $q \in F(\mathcal{S})$. Thus, we compute that

$$
\begin{aligned}
\left\|x_{n+1}-q\right\|= & \left\|\alpha_{n} f\left(x_{n}\right)+\beta_{n} x_{n}+\left(1-\alpha_{n}-\beta_{n}\right) \frac{1}{s_{n}} \int_{0}^{s_{n}} T(s) x_{n} d s-q\right\| \\
\leq & \alpha_{n}\left\|f\left(x_{n}\right)-q\right\|+\beta_{n}\left\|x_{n}-q\right\|+\left(1-\alpha_{n}-\beta_{n}\right)\left\|\frac{1}{s_{n}} \int_{0}^{s_{n}} T(s) x_{n} d s-q\right\| \\
\leq & \alpha_{n}\left(\left\|f\left(x_{n}\right)-f(q)\right\|+\|f(q)-q\|\right)+\beta_{n}\left\|x_{n}-q\right\| \\
& +\left(1-\alpha_{n}-\beta_{n}\right) \frac{1}{s_{n}} \int_{0}^{s_{n}}\left\|T(s) x_{n}-q\right\| d s \\
\leq & \alpha_{n} \alpha\left\|x_{n}-q\right\|+\alpha_{n}\|f(q)-q\|+\left(1-\alpha_{n}\right)\left\|x_{n}-q\right\| \\
= & \left(1-\alpha_{n}(1-\alpha)\right)\left\|x_{n}-q\right\|+\alpha_{n}\|f(q)-q\| \\
\leq & \max \left\{\left\|x_{n}-q\right\|, \frac{1}{1-\alpha}\|f(q)-q\|\right\} \\
& \vdots \\
\leq & \max \left\{\left\|x_{0}-q\right\|, \frac{1}{1-\alpha}\|f(q)-q\|\right\} .
\end{aligned}
$$

Therefore, $\left\{x_{n}\right\}$ is bounded, $\left\{f\left(x_{n}\right)\right\}$ and $\left\{T(s) x_{n}\right\}$ for every $0 \leq s<\infty$ are also bounded.

Next we show $\left\|x_{n}-T(h) x_{n}\right\| \rightarrow 0$ as $n \rightarrow \infty$. Notice that

$$
\begin{aligned}
\left\|x_{n+1}-T(h) x_{n+1}\right\| \leq & \left\|x_{n+1}-\frac{1}{s_{n}} \int_{0}^{s_{n}} T(s) x_{n} d s\right\| \\
& +\left\|\frac{1}{s_{n}} \int_{0}^{s_{n}} T(s) x_{n} d s-T(h)\left(\frac{1}{s_{n}} \int_{0}^{s_{n}} T(s) x_{n} d s\right)\right\| \\
& +\left\|T(h)\left(\frac{1}{s_{n}} \int_{0}^{s_{n}} T(s) x_{n} d s\right)-T(h) x_{n+1}\right\| \\
\leq & 2\left\|x_{n+1}-\frac{1}{s_{n}} \int_{0}^{s_{n}} T(s) x_{n} d s\right\| \\
& +\left\|\frac{1}{s_{n}} \int_{0}^{s_{n}} T(s) x_{n} d s-T(h)\left(\frac{1}{s_{n}} \int_{0}^{s_{n}} T(s) x_{n} d s\right)\right\| \\
\leq & 2 \alpha_{n}\left\|f\left(x_{n}\right)-\frac{1}{s_{n}} \int_{0}^{s_{n}} T(s) x_{n} d s\right\|+2 \beta_{n}\left\|x_{n}-\frac{1}{s_{n}} \int_{0}^{s_{n}} T(s) x_{n} d s\right\| \\
& +\left\|\frac{1}{s_{n}} \int_{0}^{s_{n}} T(s) x_{n} d s-T(h)\left(\frac{1}{s_{n}} \int_{0}^{s_{n}} T(s) x_{n} d s\right)\right\| .
\end{aligned}
$$

Put $z_{0}=P_{F(\mathcal{S})} x_{0}$ and $D=\left\{z \in C:\left\|z-z_{0}\right\| \leq\left\|x_{0}-z_{0}\right\|+1 /(1-\alpha)\left\|f\left(z_{0}\right)-z_{0}\right\|\right\}$. Then $D$ is a nonempty closed bounded convex subset of $C$ which is $T(s)$-invariant for each $s \in[0, \infty)$ 
and contains $\left\{x_{n}\right\}$. So without loss of generality, we may assume that $\mathcal{S}=\{T(s): 0 \leq s<\infty\}$ is a nonexpansive semigroup on $D$. By Lemma 2.4, we get

$$
\lim _{n \rightarrow \infty}\left\|\frac{1}{s_{n}} \int_{0}^{s_{n}} T(s) x_{n} d s-T(h)\left(\frac{1}{s_{n}} \int_{0}^{s_{n}} T(s) x_{n} d s\right)\right\|=0
$$

for every $h \in[0, \infty)$. On the other hand, since $\left\{x_{n}\right\},\left\{f\left(x_{n}\right)\right\}$, and $\left\{T(s) x_{n}\right\}$ are bounded, using the assumption that $\lim _{n \rightarrow \infty} \alpha_{n} \rightarrow 0, \lim _{n \rightarrow \infty} \beta_{n} \rightarrow 0$, and (4.5) into (4.4), we get that

$$
\left\|x_{n+1}-T(h) x_{n+1}\right\| \longrightarrow 0 \quad \text { as } n \longrightarrow \infty
$$

and hence

$$
\left\|x_{n}-T(h) x_{n}\right\| \longrightarrow 0 \quad \text { as } n \longrightarrow \infty \text {. }
$$

We now show that

$$
\left\langle f(\tilde{x})-\tilde{x}, J_{\varphi}\left(x_{n}-\tilde{x}\right)\right\rangle \leq 0 .
$$

Let $x_{t}=t f\left(x_{t}\right)+(1-t)\left(1 / \lambda_{t}\right) \int_{0}^{\lambda_{t}} T(s) x_{t} d s$, where $t$ and $\lambda_{t}$ satisfies the condition of Theorem 3.1. Then it follows from Theorem 3.1 that $\tilde{x}=\lim _{t \rightarrow 0} x_{t}$ and $\tilde{x}$ be the unique solution in $F(\mathcal{S})$ of the variational inequality (3.2). Clearly $\tilde{x}$ is a unique solution of (4.2). Take a subsequence $\left\{x_{n_{k}}\right\}$ of $\left\{x_{n}\right\}$ such that

$$
\limsup _{n \rightarrow \infty}\left\langle f(\tilde{x})-\tilde{x}, J_{\varphi}\left(x_{n}-\tilde{x}\right)\right\rangle=\lim _{k \rightarrow \infty}\left\langle f(\tilde{x})-\tilde{x}, J_{\varphi}\left(x_{n_{k}}-\tilde{x}\right)\right\rangle .
$$

Since $X$ is uniformly convex and hence it is reflexive, we may further assume that $x_{n_{k}} \rightarrow p$. Moreover, we note that $p \in F(\mathcal{S})$ by Lemma 2.3 and (4.7). Therefore, from (4.9) and (3.17), we have

$$
\limsup _{n \rightarrow \infty}\left\langle f(\tilde{x})-\tilde{x}, J_{\varphi}\left(x_{n}-\tilde{x}\right)\right\rangle=\left\langle f(\tilde{x})-\tilde{x}, J_{\varphi}(p-\tilde{x})\right\rangle \leq 0
$$

That is (4.8) holds. 
Finally we will show that $x_{n} \rightarrow \tilde{x}$. For each $n \geq 0$, we have

$$
\begin{aligned}
\Phi\left(\left\|x_{n+1}-\tilde{x}\right\|\right)= & \Phi\left(\left\|\alpha_{n}\left(f\left(x_{n}\right)-\tilde{x}\right)+\beta_{n}\left(x_{n}-\tilde{x}\right)+\left(1-\alpha_{n}-\beta_{n}\right)\left(\frac{1}{s_{n}} \int_{0}^{s_{n}} T(s) x_{n} d s-\tilde{x}\right)\right\|\right) \\
\leq & \Phi\left(\| \alpha_{n}\left(f\left(x_{n}\right)-f(\tilde{x})\right)+\alpha_{n}(f(\tilde{x})-\tilde{x})+\beta_{n}\left(x_{n}-\tilde{x}\right)\right. \\
& \left.+\left(1-\alpha_{n}-\beta_{n}\right)\left(\frac{1}{s_{n}} \int_{0}^{s_{n}} T(s) x_{n} d s-\tilde{x}\right) \|\right) \\
\leq & \Phi\left(\left\|\alpha_{n}\left(f\left(x_{n}\right)-f(\tilde{x})\right)+\beta_{n}\left(x_{n}-\tilde{x}\right)+\left(1-\alpha_{n}-\beta_{n}\right)\left(\frac{1}{s_{n}} \int_{0}^{s_{n}} T(s) x_{n} d s-\tilde{x}\right)\right\|\right) \\
& +\alpha_{n}\left\langle f(\tilde{x})-\tilde{x}, J_{\varphi}\left(x_{n+1}-\tilde{x}\right)\right\rangle \\
\leq & \Phi\left(\alpha_{n} \alpha\left\|x_{n}-\tilde{x}\right\|+\beta_{n}\left\|x_{n}-\tilde{x}\right\|+\left(1-\alpha_{n}-\beta_{n}\right)\left\|\frac{1}{s_{n}} \int_{0}^{s_{n}} T(s) x_{n} d s-\tilde{x}\right\|\right) \\
& +\alpha_{n}\left\langle f(\tilde{x})-\tilde{x}, J_{\varphi}\left(x_{n+1}-\tilde{x}\right)\right\rangle \\
\leq & \Phi\left(\left(1-\alpha_{n}(1-\alpha)\right)\left\|x_{n}-\tilde{x}\right\|\right)+\alpha_{n}\left\langle f(\tilde{x})-\tilde{x}, J_{\varphi}\left(x_{n+1}-\tilde{x}\right)\right\rangle \\
\leq & \left(1-\alpha_{n}(1-\alpha)\right) \Phi\left(\left\|x_{n}-\tilde{x}\right\|\right)+\alpha_{n}\left\langle f(\tilde{x})-\tilde{x}, J_{\varphi}\left(x_{n+1}-\tilde{x}\right)\right\rangle .
\end{aligned}
$$

An application of Lemma 2.6, we can obtain $\Phi\left(\left\|x_{n}-\tilde{x}\right\|\right) \rightarrow 0$, hence $\left\|x_{n}-\tilde{x}\right\| \rightarrow 0$. That is, $\left\{x_{n}\right\}$ converges strongly to a fixed point $\tilde{x}$ of $\mathcal{S}$. This completes the proof.

Theorem 4.2. Let $X$ be a uniformly convex Banach space with a uniformly Gâteaux differentiable norm and $C$ be a nonempty closed convex subset of $X$. Let $S=\{T(s): 0 \leq s<\infty\}$ be a nonexpansive semigroup from $C$ into itself such that $F(\mathcal{S})=\bigcap_{s>0} F(T(s)) \neq \emptyset$ and $f: C \rightarrow C$ a contraction mapping with the contractive coefficient $\alpha \in[0,1)$. Let $\left\{\alpha_{n}\right\}$ and $\left\{\beta_{n}\right\}$ be the sequence in $(0,1)$ which satisfies $\alpha_{n}+\beta_{n}<1, \lim _{n \rightarrow \infty} \alpha_{n} \rightarrow 0, \lim _{n \rightarrow \infty} \beta_{n} \rightarrow 0$, and $\sum_{n=1}^{\infty} \alpha_{n}=\infty$, and $\left\{s_{n}\right\}$ is a positive real divergent sequence such that $\lim _{n \rightarrow \infty} s_{n} \rightarrow \infty$. If the sequence $\left\{x_{n}\right\}$ defined by $x_{0} \in C$ and

$$
x_{n+1}=\alpha_{n} f\left(x_{n}\right)+\beta_{n} x_{n}+\left(1-\alpha_{n}-\beta_{n}\right) \frac{1}{s_{n}} \int_{0}^{s_{n}} T(s) x_{n} d s, \quad n \geq 0
$$

Then $\left\{x_{n}\right\}$ converges strongly to $\tilde{x}$ as $n \rightarrow \infty$, where $\tilde{x}$ is the unique solution in $F(\mathcal{S})$ of the variational inequality

$$
\langle(I-f) \tilde{x}, J(x-\tilde{x})\rangle \geq 0, \quad x \in F(\mathcal{S}) .
$$

Proof. We also show only those points in this proof which are different from that already presented in the proof of Theorem 4.1. We now show that

$$
\left\langle f(\tilde{x})-\tilde{x}, J\left(x_{n}-\tilde{x}\right)\right\rangle \leq 0 .
$$


Let $x_{t}=t f\left(x_{t}\right)+(1-t)\left(1 / \lambda_{t}\right) \int_{0}^{\lambda_{t}} T(s) x_{t} d s$, where $t$ and $\lambda_{t}$ satisfies the condition of Theorem 3.2. Then it follows from Theorem 3.2 that $\tilde{x}=\lim _{t \rightarrow 0} x_{t}$ and $\tilde{x}$ is the unique solution in $F(\mathcal{S})$ of the variational inequality (3.23). Clearly $\tilde{x}$ is a unique solution of (4.13). Take a subsequence $\left\{x_{n_{k}}\right\}$ of $\left\{x_{n}\right\}$ such that

$$
\limsup _{n \rightarrow \infty}\left\langle f(\tilde{x})-\tilde{x}, J\left(x_{n}-\tilde{x}\right)\right\rangle=\lim _{k \rightarrow \infty}\left\langle f(\tilde{x})-\tilde{x}, J\left(x_{n_{k}}-\tilde{x}\right)\right\rangle .
$$

Since $X$ is uniformly convex and hence it is reflexive, we may further assume that $x_{n_{k}} \rightarrow p$. Moreover, we note that $p \in F(\mathcal{S})$ by Lemma 2.3 and (4.7). Therefore, from (4.15) and (3.23), we have

$$
\limsup _{n \rightarrow \infty}\left\langle f(\tilde{x})-\tilde{x}, J\left(x_{n}-\tilde{x}\right)\right\rangle=\langle f(\tilde{x})-\tilde{x}, J(p-\tilde{x})\rangle \leq 0 .
$$

That is, (4.14) holds.

Finally we will show that $x_{n} \rightarrow \tilde{x}$. For each $n \geq 0$, by Lemma 2.2, we have

$$
\begin{aligned}
\left\|x_{n+1}-\tilde{x}\right\|^{2}= & \left\|\alpha_{n}\left(f\left(x_{n}\right)-\tilde{x}\right)+\beta_{n}\left(x_{n}-\tilde{x}\right)+\left(1-\alpha_{n}-\beta_{n}\right)\left(\frac{1}{s_{n}} \int_{0}^{s_{n}} T(s) x_{n} d s-\tilde{x}\right)\right\|^{2} \\
\leq & \left\|\left(1-\alpha_{n}-\beta_{n}\right)\left(\frac{1}{s_{n}} \int_{0}^{s_{n}} T(s) x_{n} d s-\tilde{x}\right)+\beta_{n}\left(x_{n}-\tilde{x}\right)\right\|^{2} \\
& +2 \alpha_{n}\left\langle f\left(x_{n}\right)-\tilde{x}, J\left(x_{n+1}-\tilde{x}\right)\right\rangle \\
\leq & \left(\left(1-\alpha_{n}-\beta_{n}\right)\left\|\frac{1}{s_{n}} \int_{0}^{s_{n}} T(s) x_{n} d s-\tilde{x}\right\|+\beta_{n}\left\|x_{n}-\tilde{x}\right\|\right)^{2} \\
& +2 \alpha_{n}\left\langle f\left(x_{n}\right)-\tilde{x}, J\left(x_{n+1}-\tilde{x}\right)\right\rangle \\
\leq & \left(\left(1-\alpha_{n}\right)\left\|x_{n}-\tilde{x}\right\|\right)^{2}+2 \alpha_{n}\left\langle f\left(x_{n}\right)-f(\tilde{x}), J\left(x_{n+1}-\tilde{x}\right)\right\rangle \\
& +2 \alpha_{n}\left\langle f(\tilde{x})-\tilde{x}, J\left(x_{n+1}-\tilde{x}\right)\right\rangle \\
\leq & \left(1-\alpha_{n}\right)^{2}\left\|x_{n}-\tilde{x}\right\|^{2}+2 \alpha_{n}\left\|f\left(x_{n}\right)-f(\tilde{x})\right\|\left\|J\left(x_{n+1}-\tilde{x}\right)\right\| \\
& +2 \alpha_{n}\left\langle f(\tilde{x})-\tilde{x}, J\left(x_{n+1}-\tilde{x}\right)\right\rangle \\
\leq & \left(1-\alpha_{n}\right)^{2}\left\|x_{n}-\tilde{x}\right\|^{2}+2 \alpha_{n} \alpha\left\|x_{n}-\tilde{x}\right\|\left\|x_{n+1}-\tilde{x}\right\|+2 \alpha_{n}\left\langle f(\tilde{x})-\tilde{x}, J\left(x_{n+1}-\tilde{x}\right)\right\rangle \\
\leq & \left(1-\alpha_{n}\right)^{2}\left\|x_{n}-\tilde{x}\right\|^{2}+\alpha_{n} \alpha\left(\left\|x_{n}-\tilde{x}\right\|^{2}+\left\|x_{n+1}-\tilde{x}\right\|^{2}\right)+2 \alpha_{n}\left\langle f(\tilde{x})-\tilde{x}, J\left(x_{n+1}-\tilde{x}\right)\right\rangle \\
= & \left(\left(1-\alpha_{n}\right)^{2}+\alpha_{n} \alpha\right)\left\|x_{n}-\tilde{x}\right\|^{2}+\alpha_{n} \alpha\left\|x_{n+1}-\tilde{x}\right\|^{2}+2 \alpha_{n}\left\langle f(\tilde{x})-\tilde{x}, J\left(x_{n+1}-\tilde{x}\right)\right\rangle,
\end{aligned}
$$


which implies that

$$
\begin{aligned}
\left\|x_{n+1}-\tilde{x}\right\|^{2} \leq & \frac{1-2 \alpha_{n}+\alpha_{n}^{2}+\alpha_{n} \alpha}{1-\alpha_{n} \alpha}\left\|x_{n}-\tilde{x}\right\|^{2}+\frac{2 \alpha_{n}}{1-\alpha_{n} \alpha}\left\langle f(\tilde{x})-\tilde{x}, J\left(x_{n+1}-\tilde{x}\right)\right\rangle \\
= & {\left[1-\frac{2(1-\alpha) \alpha_{n}}{1-\alpha_{n} \alpha}\right]\left\|x_{n}-\tilde{x}\right\|^{2}+\frac{\alpha_{n}^{2}}{1-\alpha_{n} \alpha}\left\|x_{n}-\tilde{x}\right\|^{2}+\frac{2 \alpha_{n}}{1-\alpha_{n} \alpha}\left\langle f(\tilde{x})-\tilde{x}, J\left(x_{n+1}-\tilde{x}\right)\right\rangle } \\
\leq & {\left[1-\frac{2(1-\alpha) \alpha_{n}}{1-\alpha_{n} \alpha}\right]\left\|x_{n}-\tilde{x}\right\|^{2} } \\
& +\frac{2(1-\alpha) \alpha_{n}}{1-\alpha_{n} \alpha}\left\{\frac{\alpha_{n} M}{2(1-\alpha)}+\frac{1}{1-\alpha}\left\langle f(\tilde{x})-\tilde{x}, J\left(x_{n+1}-\tilde{x}\right)\right\rangle\right\} \\
= & \left(1-\delta_{n}\right)\left\|x_{n}-\tilde{x}\right\|^{2}+\delta_{n} \gamma_{n},
\end{aligned}
$$

where $M=\sup \left\{\left\|x_{n}-\tilde{x}\right\|^{2}: n \in \mathbb{N}\right\}, \delta_{n}:=2(1-\alpha) \alpha_{n} /\left(1-\alpha_{n} \alpha\right)$, and $\gamma_{n}:=\left(\alpha_{n} M / 2(1-\right.$ $\alpha))+(1 /(1-\alpha))\left\langle f(\tilde{x})-\tilde{x}, J\left(x_{n+1}-\tilde{x}\right)\right\rangle$. It is easily to see that $\delta_{n} \rightarrow 0, \sum_{n=1}^{\infty} \delta_{n}=\infty$ and $\lim \sup _{n \rightarrow \infty} \gamma_{n} \leq 0$ by (4.14). Finally by using Lemma 2.6, we can obtain $\left\{x_{n}\right\}$ converges strongly to a fixed point $\tilde{x} \in F(\mathcal{S})$. This completes the proof.

\section{Applications}

Theorem 5.1. Let $X$ be a uniformly convex Banach space that has a weakly continuous duality map $J_{\varphi}$ with gauge $\varphi$ and $C$ be a nonempty closed convex subset of $X$. Let $S=\{T(s): 0 \leq s<\infty\}$ be a nonexpansive semigroup from $C$ into itself such that $F(\mathcal{S})=\bigcap_{s>0} F(T(s)) \neq \emptyset$ and $f: C \rightarrow C$ a contraction mapping with the contractive coefficient $\alpha \in[0,1)$. Let $\left\{\alpha_{n}\right\}$ be the sequence in $(0,1)$ which satisfies $\lim _{n \rightarrow \infty} \alpha_{n} \rightarrow 0$ and $\sum_{n=1}^{\infty} \alpha_{n}=\infty$, and $\left\{s_{n}\right\}$ is a positive real divergent sequence such that $\lim _{n \rightarrow \infty} s_{n} \rightarrow \infty$. If the sequence $\left\{x_{n}\right\}$ defined by $x_{0} \in C$ and

$$
x_{n+1}=\alpha_{n} f\left(x_{n}\right)+\left(1-\alpha_{n}\right) \frac{1}{s_{n}} \int_{0}^{s_{n}} T(s) x_{n} d s, \quad n \geq 0 .
$$

Then $\left\{x_{n}\right\}$ converges strongly to $\tilde{x}$ as $n \rightarrow \infty$, where $\tilde{x}$ is the unique solution in $F(\mathcal{S})$ of the variational inequality

$$
\langle(I-f) \tilde{x}, J(x-\tilde{x})\rangle \geq 0, \quad x \in F(\mathcal{S}) .
$$

Proof. Taking $\beta_{n}=0$ in the in Theorem 4.1, we get the desired conclusion easily.

Theorem 5.2. Let $X$ be a uniformly convex Banach space with a uniformly Gateaux differentiable norm and $C$ be a nonempty closed convex subset of X. Let $\mathcal{S}=\{T(s): 0 \leq s<\infty\}$ be a nonexpansive semigroup from $C$ into itself such that $F(S)=\bigcap_{s>0} F(T(s)) \neq \emptyset$ and $f: C \rightarrow C a$ contraction mapping with the contractive coefficient $\alpha \in[0,1)$. Let $\left\{\alpha_{n}\right\}$ be the sequence in $(0,1)$ 
which satisfies $\lim _{n \rightarrow \infty} \alpha_{n} \rightarrow 0$ and $\sum_{n=1}^{\infty} \alpha_{n}=\infty$, and $\left\{s_{n}\right\}$ is a positive real divergent sequence such that $\lim _{n \rightarrow \infty} s_{n} \rightarrow \infty$. If the sequence $\left\{x_{n}\right\}$ defined by $x_{0} \in C$ and

$$
x_{n+1}=\alpha_{n} f\left(x_{n}\right)+\left(1-\alpha_{n}\right) \frac{1}{s_{n}} \int_{0}^{s_{n}} T(s) x_{n} d s, \quad n \geq 0 .
$$

Then $\left\{x_{n}\right\}$ converges strongly to $\tilde{x}$ as $n \rightarrow \infty$, where $\tilde{x}$ is the unique solution in $F(\mathcal{S})$ of the variational inequality

$$
\langle(I-f) \tilde{x}, J(x-\tilde{x})\rangle \geq 0, \quad x \in F(\mathcal{S}) .
$$

Proof. Taking $\beta_{n}=0$ in the in Theorem 4.2, we get the desired conclusion easily.

When $X$ is a Hilbert space, we can get the following corollary easily.

Corollary 5.3 (Reich [2]). Let $C$ be a nonempty closed convex subset of a real Hilbert space H. Let $\mathcal{S}=\{T(s): 0 \leq s<\infty\}$ be a strongly continuous semigroup of nonexpansive mapping on $C$ such that $F(S)$ is nonempty. Let $\left\{\alpha_{n}\right\}$ and $\left\{\beta_{n}\right\}$ be sequences of real numbers in $(0,1)$ which satisfies $\alpha_{n}+\beta_{n}<1$, $\lim _{n \rightarrow \infty} \alpha_{n} \rightarrow 0, \lim _{n \rightarrow \infty} \beta_{n} \rightarrow 0$, and $\sum_{n=1}^{\infty} \alpha_{n}=\infty$. Let $f$ be a contraction of $C$ into itself with $a$ coefficient $\alpha \in[0,1)$ and $\left\{s_{n}\right\}$ be a positive real divergent sequence such that $\lim _{n \rightarrow \infty} s_{n} \rightarrow \infty$. Then the sequence $\left\{x_{n}\right\}$ defined by $x_{0} \in C$ and

$$
x_{n+1}=\alpha_{n} f\left(x_{n}\right)+\beta_{n} x_{n}+\left(1-\alpha_{n}-\beta_{n}\right) \frac{1}{s_{n}} \int_{0}^{s_{n}} T(s) x_{n} d s, \quad n \geq 0 .
$$
inequality

Then $\left\{x_{n}\right\}$ converges strongly to $\tilde{x}$, where $\tilde{x}$ is the unique solution in $F(\mathcal{S})$ of the variational

$$
\langle(I-f) \tilde{x}, x-\tilde{x}\rangle \geq 0, \quad x \in F(\mathcal{S})
$$

or equivalent $\tilde{x}=P_{F(S)}(f)(\tilde{x})$, where $P$ is a metric projection mapping from $H$ into $F(S)$.

\section{Funding}

This paper is supported by the National Science Foundation of China under Grants (10771050 and 11101305).

\section{References}

[1] F. E. Browder, "Fixed-point theorems for noncompact mappings in Hilbert space," Proceedings of the National Academy of Sciences of the United States of America, vol. 53, pp. 1272-1276, 1965.

[2] S. Reich, "Strong convergence theorems for resolvents of accretive operators in Banach spaces," Journal of Mathematical Analysis and Applications, vol. 75, no. 1, pp. 287-292, 1980.

[3] F. E. Browder, "Convergence of approximants to fixed points of nonexpansive non-linear mappings in Banach spaces," Archive for Rational Mechanics and Analysis, vol. 24, pp. 82-90, 1967.

[4] Y. Song and S. Xu, "Strong convergence theorems for nonexpansive semigroup in Banach spaces," Journal of Mathematical Analysis and Applications, vol. 338, no. 1, pp. 152-161, 2008. 
[5] A. Moudafi, "Viscosity approximation methods for fixed-points problems," Journal of Mathematical Analysis and Applications, vol. 241, no. 1, pp. 46-55, 2000.

[6] H.-K. Xu, "Viscosity approximation methods for nonexpansive mappings," Journal of Mathematical Analysis and Applications, vol. 298, no. 1, pp. 279-291, 2004.

[7] S. Plubtieng and R. Punpaeng, "Fixed-point solutions of variational inequalities for nonexpansive semigroups in Hilbert spaces," Mathematical and Computer Modelling, vol. 48, no. 1-2, pp. 279-286, 2008.

[8] R. Chen and Y. Song, "Convergence to common fixed point of nonexpansive semigroups," Journal of Computational and Applied Mathematics, vol. 200, no. 2, pp. 566-575, 2007.

[9] W. Takahashi, Nonlinear Functional Analysis-Fixed Point Theory and Its Applications, Yokohama Publishers, Yokohama, Japan, 2000.

[10] K. Goebel and S. Reich, Uniform Convexity, Hyperbolic Geometry, and Nonexpansive Mappings, vol. 83 of Monographs and Textbooks in Pure and Applied Mathematics, Marcel Dekker, New York, NY, USA, 1984.

[11] H.-K. Xu, "Strong convergence of an iterative method for nonexpansive and accretive operators," Journal of Mathematical Analysis and Applications, vol. 314, no. 2, pp. 631-643, 2006.

[12] F. E. Browder, "Semicontractive and semiaccretive nonlinear mappings in Banach spaces," Bulletin of the American Mathematical Society, vol. 74, pp. 660-665, 1968.

[13] F. E. Browder, "Convergence theorems for sequences of nonlinear operators in Banach spaces," Mathematische Zeitschrift, vol. 100, pp. 201-225, 1967.

[14] W. Takahashi and Y. Ueda, "On Reich's strong convergence theorems for resolvents of accretive operators," Journal of Mathematical Analysis and Applications, vol. 104, no. 2, pp. 546-553, 1984. 


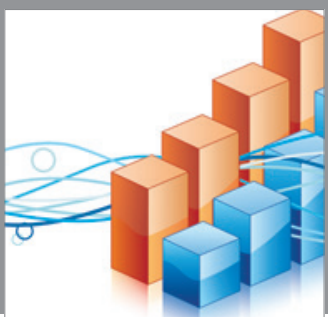

Advances in

Operations Research

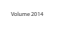

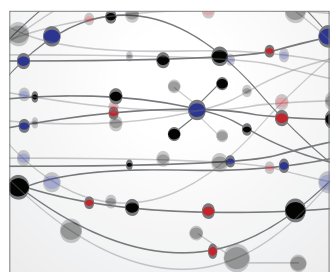

\section{The Scientific} World Journal
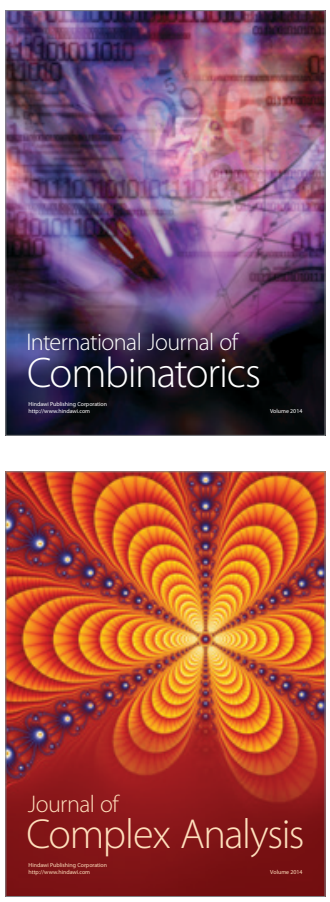

International Journal of

Mathematics and

Mathematical

Sciences
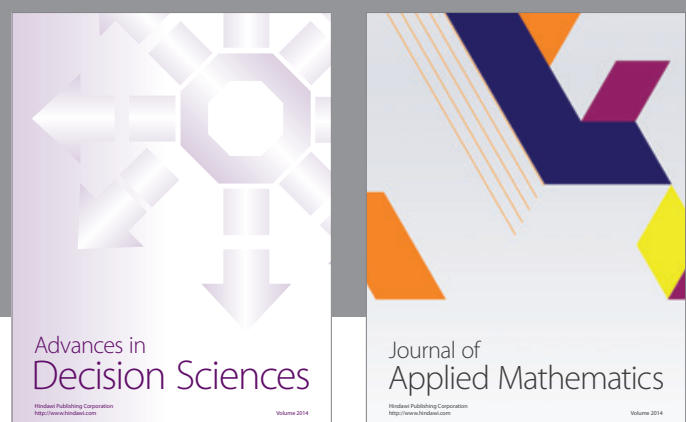

Journal of

Applied Mathematics
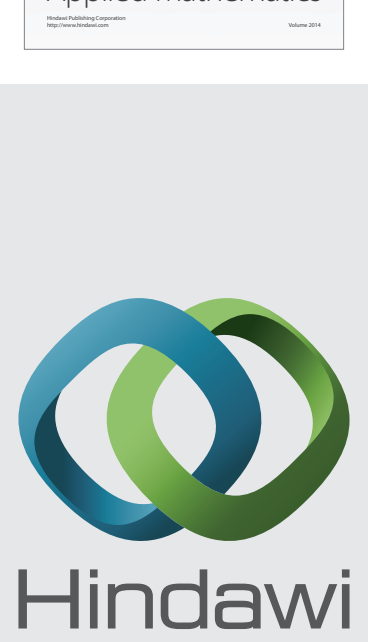

Submit your manuscripts at http://www.hindawi.com
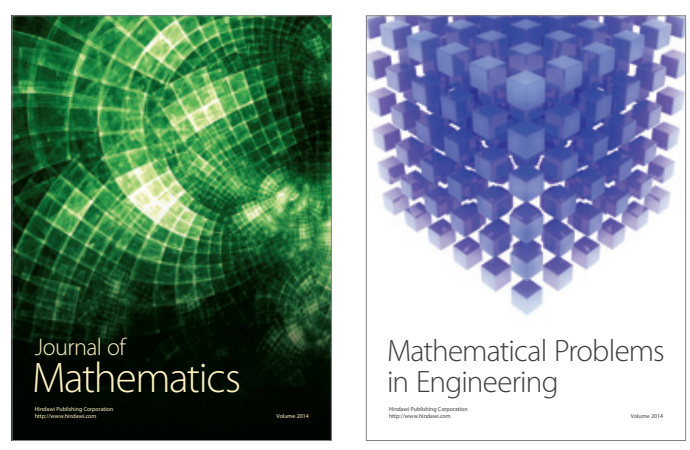

Mathematical Problems in Engineering
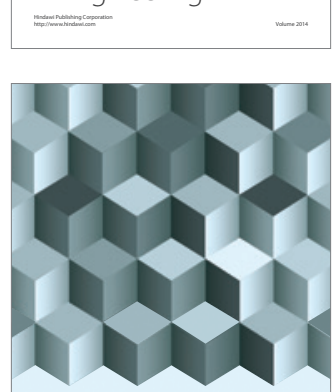

Journal of

Function Spaces
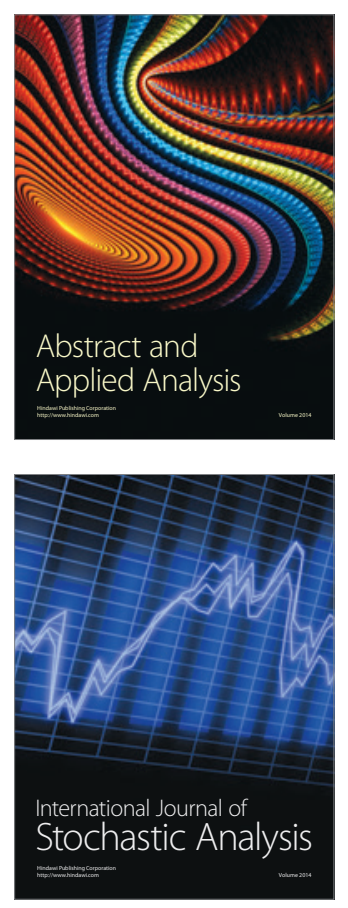

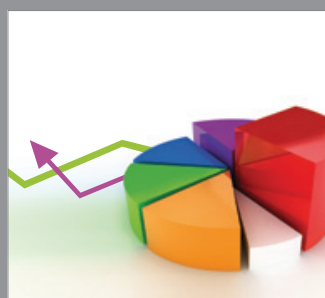

ournal of

Probability and Statistics

Promensencen
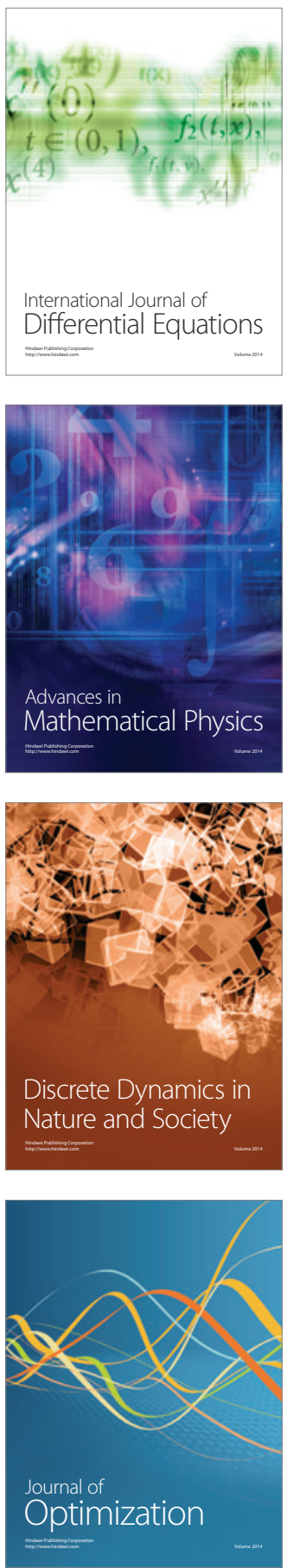